\title{
A New Approach For the Ranking of Fuzzy Sets With Different Heights
}

\author{
Pushpinder Singh \\ School of Mathematics and Computer Applications \\ Thapar University, Patiala-147 004 \\ India \\ pushpindersnl@gmail.com
}

\begin{abstract}
Ranking of fuzzy sets plays an important role in decision making, optimization, forecasting, etc. Fuzzy sets must be ranked before an action is taken by a decision maker. Fuzzy sets with different heights are a generalization of the ordinary fuzzy sets. In this paper, with the help of several counterexamples, it is proved that the ranking method proposed by Lee and Chen (Expert Systems with Applications 34, 2008, 2763-2771) is incorrect. The main aim of this paper is to propose a new approach for the ranking of fuzzy sets with different heights. The main advantage of the proposed approach is that with it the correct ordering of fuzzy sets with different heights, and also the results of the proposed ranking method and the existing ranking method, can be compared.
\end{abstract}

Keywords: sets, fuzzy sets with different heights, ranking function.

\section{Introduction}

Fuzzy set theory [24] is a powerful tool to deal with real-life situations. Real numbers can be linearly ordered by $\leq$ or $\geq$; however, this type of inequality does not exist in fuzzy numbers. Since fuzzy numbers are represented by possibility distribution, they can overlap with each other and it is difficult to determine clearly whether one fuzzy number is larger or smaller than the other. An efficient approach for ordering the fuzzy numbers is by using a ranking function $\mathfrak{R}: F(R) \rightarrow R$, where $F(R)$ is a set of fuzzy numbers defined on the real line which maps each fuzzy number into the real line, where a natural order exists. Thus, specific ranking of fuzzy numbers is an important procedure for decision making in a fuzzy environment and, generally, has become one of the main problems in fuzzy set theory.

The method for ranking was first proposed by Jain [14]. Yager [23] proposed four indices that may be employed for the purpose of ordering fuzzy quantities in [0, 1]. Campos and Gonzalez [4] proposed a subjective approach for ranking of fuzzy numbers. Liou and Wang [17] developed a ranking method based on the integral value index. Cheng [10] presented a method for ranking fuzzy numbers by using the distance method. Kwang and Lee [15] considered the overall possibility distributions of fuzzy numbers in their evaluations and proposed a ranking method. Modarres and Sadi-Nezhad [18] proposed a ranking method based on the preference function which measures the fuzzy numbers point by point and, at each point, the most preferred number is identified. Chu and Tsao [11] proposed a method for ranking fuzzy numbers with the area between the centroid point and the original point. Deng and Liu [12] presented a centroid index method for ranking fuzzy numbers. Chen and Chen [5] presented a method for ranking generalized trapezoidal fuzzy numbers. Wang and Lee [22] used the centroid concept in developing their ranking index.

Chen and Tang [8] proposed a method for ranking nonnormal p-norm trapezoidal fuzzy numbers. Lee and Chen [16] presented a new method for ranking fuzzy sets and used the proposed fuzzy ranking method to present a new fuzzy risk analysis algorithm to deal with fuzzy risk analysis problems. Chen and Wang [9] studied the fuzzy risk analysis based on the ranking of fuzzy numbers. Abbasbandy and Hajjari [1] introduced a new approach for ranking trapezoidal fuzzy numbers based on the left and right spreads at some levels of trapezoidal fuzzy numbers. Chen and Chen [6] presented a method for fuzzy risk analysis based on of ranking generalized fuzzy numbers with different 
heights and different spreads. Ramli and Mohamad [19] presented the comprehensive survey of different methods for the ranking of fuzzy numbers. Asady [2] indicated the shortcomings of Wang method and proposed a revised method in which shortcomings for ranking fuzzy numbers are removed. Assady [3] suggested an interesting approach to crisp function approximation of fuzzy numbers and define the epsilon - neighborhood of the fuzzy number. The method leads to the crisp function which is the best one related to a certain measure of distance between the fuzzy number and a crisp function of the set support function. Chen et al. [7] presented a new method for fuzzy risk analysis based on the proposed new fuzzy ranking method for ranking generalized fuzzy numbers with different left heights and right heights. Also, they proposed a new method for fuzzy risk analysis based on the proposed fuzzy ranking method. Ezzati et al. [13] modified the method of Abbasbandy and Hajjari [1].

In this paper, with the help of counterexamples, it is shown that the ranking approach proposed by Lee and Chen [16] is incorrect. A new approach is proposed for the ranking of fuzzy sets with different heights. Besides, the results of the proposed approach and the existing ranking approach are compared.

This paper is organized as follows: In Section 2, some basic definitions and arithmetic operations between fuzzy sets with different heights are presented. In Section 3, Lee and Chen's [16] ranking approach is reviewed. Shortcomings of Lee and Chen's approach [16] are pointed out in Section 4. In Section 5, a new approach is proposed for the ranking of fuzzy sets with different heights. Results of the proposed approach and the existing ranking approach are compared in Section 6 . The final section is for conclusions.

\section{Preliminaries}

In this section some basic definitions and arithmetic operations are presented.

\subsection{Basic definitions}

Definition 1 [24.] The characteristic function $\mu_{A}$ of a crisp set $A \subseteq X$ assigns a value, either 0 or 1 , to each member in $X$. This function can be generalized to a function $\mu_{\tilde{A}}$ such that the value assigned to the element of the universal set $X$ fall within a specified range i.e. $\mu_{\tilde{A}}: X \rightarrow[0,1]$. The assigned value indicates the membership grade of the element in the set $A$.

The function $\mu_{\tilde{A}}$ is called the membership function, and the set $\tilde{A}=\left\{\left(x, \mu_{\tilde{A}}(x)\right) ; x \in X\right\}$, defined by $\mu_{\tilde{A}}(x)$ for each $x \in X$, is called a fuzzy set.

Definition 2 [16]. A fuzzy set $\widetilde{A}_{i}=\left(a_{i}, b_{i}, c_{i}, d_{i} ; L_{i H}, R_{i H}\right), 1 \leq i \leq n$ is said to be a fuzzy set with different height if its membership function is given by

$$
\mu_{\tilde{A}_{i}}(x)=\left\{\begin{array}{lc}
0, & -\infty<x \leq a_{i} \\
L_{i H}\left(\frac{x-a_{i}}{b_{i}-a_{i}}\right), & a_{i} \leq x<b_{i} \\
L_{i H}\left(\frac{c_{i}-x}{c_{i}-b_{i}}\right)+R_{i H}\left(\frac{x-b_{i}}{c_{i}-b_{i}}\right), & b_{i} \leq x \leq c_{i} \\
R_{i H}\left(\frac{x-d_{i}}{c_{i}-d_{i}}\right), & c_{i} \leq x<d_{i} \\
0, & d_{i} \leq x \leq \infty
\end{array}\right.
$$

where $L_{i H}$ and $R_{i H}$ denotes the left and right height of fuzzy sets with different heights, respectively.

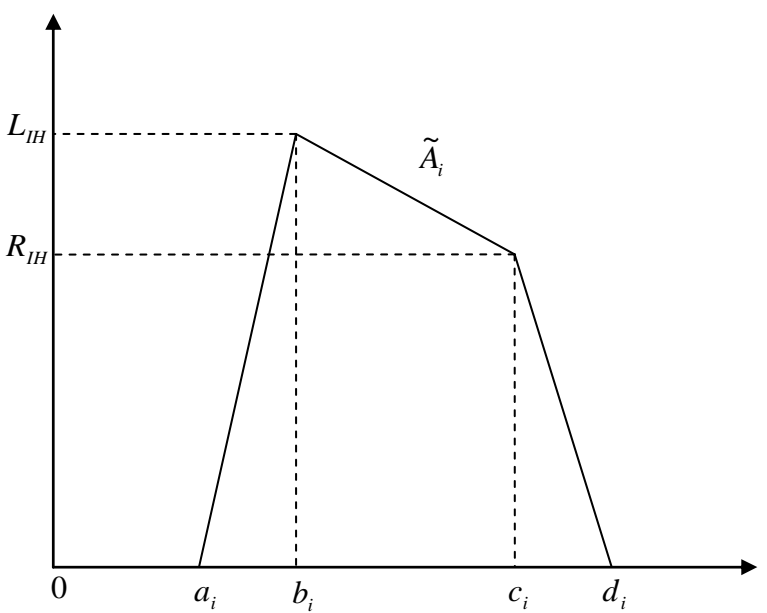

Figure 1. Fuzzy set with different height. 


\subsection{Arithmetic Operations}

Let $\quad \tilde{A}_{1}=\left(a_{1}, b_{1}, c_{1}, d_{1} ; L_{1 H}, R_{1 H}\right) \quad$ and $\tilde{A}_{2}=\left(a_{2}, b_{2}, c_{2}, d_{2} ; L_{2 H}, R_{2 H}\right)$ be two fuzzy sets with different heights [16] then

$$
\begin{aligned}
& \tilde{A}_{1} \oplus \tilde{A}_{2}=\left(a_{1}+a_{2}, b_{1}+b_{2}, c_{1}+c_{2}, d_{1}+d_{2} ;\right. \\
& \left.\min \left(L_{1 H}, L_{2 H}\right), \min \left(R_{1 H}, R_{2 H}\right)\right) \\
& \tilde{A}_{1} \Theta \tilde{A}_{2}=\left(a_{1}-d_{2}, b_{1}-c_{2}, c_{1}-b_{2}, d_{1}-a_{2} ;\right. \\
& \left.\min \left(L_{1 H}, L_{2 H}\right), \min \left(R_{1 H}, R_{2 H}\right)\right)
\end{aligned}
$$

$$
\lambda \tilde{A}_{1}= \begin{cases}\left(\lambda a_{1}, \lambda b_{1}, \lambda c_{1}, \lambda d_{1} ; L_{1 H}, R_{1 H}\right) & \lambda \geq 0 \\ \left(\lambda d_{1}, \lambda c_{1}, \lambda b_{1}, \lambda a_{1} ; L_{1 H}, R_{1 H}\right)_{p} & \lambda<0\end{cases}
$$

\section{Lee and Chen's ranking approach}

Let $\tilde{A}_{1}, \tilde{A}_{2}, \ldots$, and $\tilde{A}_{n}$ be a set of trapezoidal fuzzy numbers, where

$\tilde{A}_{i}=\left(a_{i 1}, a_{i 2}, a_{i 3}, a_{i 4} ; L_{i H}, R_{i H}\right), \quad 1 \leq i \leq n$. The proposed method for ranking fuzzy numbers is now presented as follows:

Step 1. Find the values of $R_{i H}, L_{i H}, R_{i M}, L_{i M}$, $R_{i s}, L_{i s}$ and $T_{i s}$ of each fuzzy number $\tilde{A}_{i}$, where

$R_{i H}$ denotes the right height of the fuzzy number $\tilde{A}_{i}$.

$L_{i H}$ denotes the left height of the fuzzy number $\tilde{A}_{i}$.

$R_{i M}=\frac{\left(a_{i 3}+a_{i 4}\right)}{2}$ denotes the average of the right elements $a_{i 3}$ and $a_{i 4}$.
$L_{i M}=\frac{\left(a_{i 1}+a_{i 2}\right)}{2}$ denotes the average of the left elements $a_{i 1}$ and $a_{i 2}$.

$R_{i S}=\sqrt{\frac{1}{2} \sum_{j=3}^{4}\left(a_{i j}-\frac{1}{2} \sum_{j=3}^{4} a_{i j}\right)^{2}} \quad$ denotes the standard deviation of the right elements $a_{i 3}$ and $a_{i 4}$.

$L_{i S}=\sqrt{\frac{1}{2} \sum_{j=1}^{2}\left(a_{i j}-\frac{1}{2} \sum_{j=1}^{2} a_{i j}\right)^{2}} \quad$ denotes the standard deviation of the right elements $a_{i 1}$ and $a_{i 2}$.

$T_{i S}=\sqrt{\frac{1}{4} \sum_{j=1}^{4}\left(a_{i j}-\frac{1}{4} \sum_{j=1}^{4} a_{i j}\right)^{2}}$ denotes the standard deviation of the elements $a_{i 1}, a_{i 2}, a_{i 3}, a_{i 4}$ and $1 \leq i \leq n$.

Step 2. Choose two proper values for $\alpha$ and $\beta$, where $\alpha$ denotes the expert's degree of confidence, $\alpha \in[0,1], \beta$ denotes the index of optimism of the decision maker and $\beta \in[0,1]$. In general, let $\alpha=0.5$ and $\beta=0.5$ be proper values for ranking fuzzy numbers.

Step 3. Calculate the ranking values Rank $\left(\tilde{A}_{i}\right)$ of each fuzzy number $\tilde{A}_{i}$ as follows:

Rank

$$
\begin{aligned}
& \left(\widetilde{A}_{i}\right)=\alpha\left[\beta R_{i H}\left(\widetilde{A}_{i}\right)+(1-\beta) L_{i H}\left(\tilde{A}_{i}\right)\right]+(1-\alpha)\left[\beta R_{i M}\left(\tilde{A}_{i}\right)+\right. \\
& \left.\left.(1-\beta) L_{i M}\left(\tilde{A}_{i}\right)\right]-\frac{1}{3}\left(\beta R_{i s}\left(\tilde{A}_{i}\right)+(1-\beta) L_{i s}\left(\tilde{A}_{i}\right)+T_{i s}\left(\tilde{A}_{i}\right)\right)\right]
\end{aligned}
$$

The larger the ranking value Rank $\left(\tilde{A}_{i}\right)$, the better the ranking of the fuzzy number $\widetilde{A}_{i}$. 


\section{Shortcomings of Lee and Chen's ranking approach}

Wang and Keere [21] proposed the following reasonable properties for the validation of any ranking function:

If $\widetilde{A}$ and $\widetilde{B}$ are normal fuzzy sets then

$$
\begin{aligned}
& \tilde{A} \succ \tilde{B} \Rightarrow(\tilde{A} \oplus \tilde{C}) \succ(\tilde{B} \oplus \tilde{C}) \\
& \tilde{A} \prec \tilde{B} \Rightarrow(\tilde{A} \oplus \tilde{C}) \prec(\tilde{B} \oplus \tilde{C}) \\
& \tilde{A}: \tilde{B} \Rightarrow(\tilde{A} \oplus \tilde{C}):(\tilde{B} \oplus \tilde{C})
\end{aligned}
$$

where, $\widetilde{C}$ is normal fuzzy set.

For the fuzzy sets with different heights, the same property can be written as

If $\tilde{A}=\left(a_{1}, b_{1}, c_{1}, d_{1} ; L_{1 H}, R_{1 H}\right)$ and

$\widetilde{B}=\left(a_{2}, b_{2}, c_{2}, d_{2} ; L_{2 H}, R_{2 H}\right)$ are two fuzzy sets with different heights then

$$
\begin{aligned}
& \tilde{A} \succ \widetilde{B} \Rightarrow(\tilde{A} \oplus \tilde{C}) \succ(\tilde{B} \oplus \tilde{C}) \\
& \tilde{A} \prec \tilde{B} \Rightarrow(\tilde{A} \oplus \tilde{C}) \prec(\tilde{B} \oplus \tilde{C}) \\
& \tilde{A}: \tilde{B} \Rightarrow(\tilde{A} \oplus \tilde{C}):(\tilde{B} \oplus \tilde{C})
\end{aligned}
$$

where, $\widetilde{C}=\left(a_{3}, b_{3}, c_{3}, d_{3} ; L_{3 H}, R_{3 H}\right)$ is a type-II fuzzy set and

$$
\left(L_{3 H}, R_{3 H}\right) \leq\left(\min \left(L_{1 H}, L_{2 H}\right), \min \left(R_{1 H}, R_{2 H}\right)\right) \text {. }
$$

There may exist several fuzzy sets with different heights for which the existing ranking functions [16] do not satisfy the reasonable property

$\tilde{A} \succ \widetilde{B} \Rightarrow \tilde{A} \oplus \tilde{C} \succ \widetilde{B} \oplus \widetilde{C}$, i.e., according to existing ranking approaches

$\widetilde{A} \succ \widetilde{B} \nRightarrow \widetilde{A} \oplus \widetilde{C} \succ \widetilde{B} \oplus \widetilde{C}$ which is a contradiction according to Wang and Keere [21].
Example 1. Let $\tilde{A}=(1,2,3,4 ; 0.6,0.4)$, $\widetilde{B}=(0,3,4,5 ; 0.4,0.2)$ and $\widetilde{C}=(1,3,4,5 ; 0.4,0.2)$ be three fuzzy sets with different heights. Then, according to existing ranking approaches [16], $\widetilde{A} \prec \widetilde{B}$ but $\tilde{A} \oplus \widetilde{C} \succ \widetilde{B} \oplus \widetilde{C}$ i.e., , which is a contradiction.

Example 2. Let $\quad \tilde{A}=(2,5,6,7 ; 0.6,0.4)$, $\widetilde{B}=(3,4,5,6 ; 0.8,0.6)$ and $\widetilde{C}=(4,3,7,8 ; 0.6,0.4)$ be three fuzzy sets with different heights. Then, according to existing ranking approaches [16], $\tilde{A} \succ \widetilde{B}$ but $\tilde{A} \oplus \widetilde{C} \prec \widetilde{B} \oplus \widetilde{C}$ i.e., , which is a contradiction.

\section{A new approach for the ranking of fuzzy sets with different heights}

In this section, a new approach is proposed for the ranking of fuzzy sets with different heights. To overcome the shortcomings discussed in Section 4 , the following definitions are proposed.

Definition 3. For any fuzzy set with different height $\tilde{A}_{i}=\left(a_{i}, b_{i}, c_{i}, d_{i} ; L_{i H}, R_{i H}\right)$, the expectation value of centroid is defined as follows:

$$
M_{i}=\frac{\int_{a_{i}}^{d_{i}} x f_{\widetilde{A}_{i}}(x)}{\int_{a_{i}}^{d_{i}} f_{\widetilde{A}_{i}}(x)}
$$

Definition 4. For any fuzzy set with different height $\tilde{A}_{i}=\left(a_{i}, b_{i}, c_{i}, d_{i} ; L_{i H}, R_{i H}\right)$, the transfer coefficient of $\tilde{A}_{i}, i=1,2, \ldots, n$, is given by

$$
\lambda_{i}=\frac{M_{i}-M_{\min }}{M_{\max }-M_{\min }}
$$

where $\quad M_{\max }=\max \left(M_{1}, M_{2}, \ldots, M_{n}\right) \quad$ and $M_{\text {min }}=\min \left(M_{1}, M_{2}, \ldots, M_{n}\right)$. 
Definition 5. Let $\widetilde{A}_{i}=\left(a_{i}, b_{i}, c_{i}, d_{i} ; L_{i H}, R_{i H}\right)$ be a fuzzy set with different height, $a_{\text {min }}=\min \left(a_{1}, a_{2}, \ldots, a_{n}\right) \quad$ and $d_{\max }=\max \left(d_{1}, d_{2}, \ldots, d_{n}\right)$. The areas $s^{L}\left(\tilde{A}_{i}\right)$ and $s^{R}\left(\tilde{A}_{i}\right)$ of the left and right side of fuzzy set with different height $\tilde{A}_{i}$ are defined as follows:

$$
\begin{aligned}
& s^{L}\left(\tilde{A}_{i}\right)=\int_{0}^{L_{i H}}\left(a+\frac{(b-a) y}{L}-a_{\min }\right) d y \\
& s^{R}\left(\tilde{A}_{i}\right)=\int_{0}^{R_{i H}}\left(d_{\max }-d-\frac{(c-d) y}{R}\right) d y
\end{aligned}
$$

From Equations 2, 3 and 4, the proposed ranking index of $\widetilde{A}_{i}, i=1,2, \ldots, n$ is defined as follows:

$$
s\left(\tilde{A}_{i}\right)=\frac{s^{L}\left(\tilde{A}_{i}\right) \lambda_{i}}{1+s^{R}\left(\tilde{A}_{i}\right)\left(1-\lambda_{i}\right)}
$$

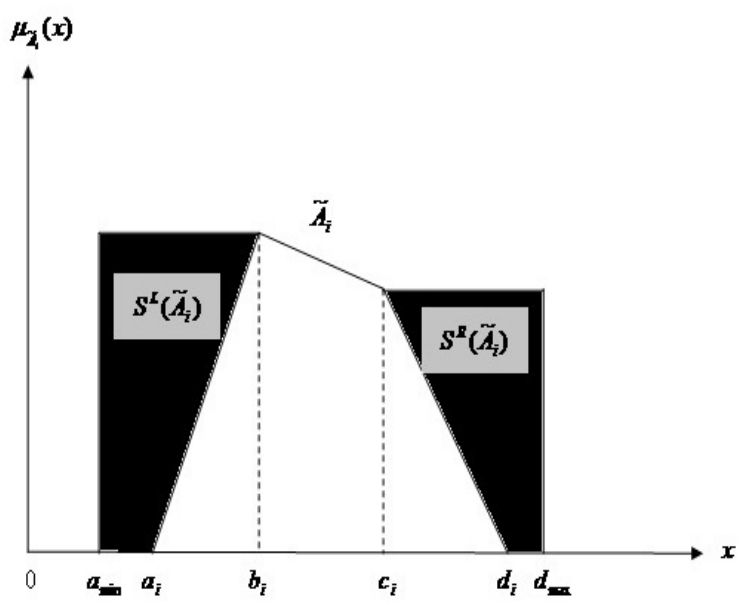

Figure 2. Fuzzy set left and right area.
Definition 6. For any two fuzzy sets with different heights, $\widetilde{A}_{i}$ and $\tilde{A}_{j}$, based on (5), their order is defined by

$$
\tilde{A}_{i} \succ \tilde{A}_{j} \text { if and only if } s\left(\tilde{A}_{i}\right) \succ s\left(\tilde{A}_{i}\right)
$$

$\tilde{A}_{i} \prec \tilde{A}_{j}$ if and only if $s\left(\tilde{A}_{i}\right) \prec s\left(\tilde{A}_{i}\right)$.

$$
\tilde{A}_{i}: \tilde{A}_{j} \text { if and only if } s\left(\tilde{A}_{i}\right): s\left(\tilde{A}_{i}\right)
$$

\subsection{Algorithm}

Let $\quad \tilde{A}_{1}=\left(a_{1}, b_{1}, c_{1}, d_{1} ; L_{1 H}, R_{1 H}\right) \quad$ and $\tilde{A}_{2}=\left(a_{2}, b_{2}, c_{2}, d_{2} ; L_{2 H}, R_{2 H}\right)$ be fuzzy sets with different heights in $F(R)$. Use the following step to compare $\tilde{A}_{1}$ and $\tilde{A}_{2}$ :

Step 1. Transform $\tilde{A}_{1}, \tilde{A}_{2}$ into $\tilde{A}_{1}^{*}$ and $\tilde{A}_{2}^{*}$ as follows:

$\tilde{A}_{1}^{*}=\left(a_{1}, b_{1}, c_{1}, d_{1} ; L_{H}, R_{H}\right)$ and

$\tilde{A}_{2}^{*}=\left(a_{2}, b_{2}, c_{2}, d_{2} ; L_{H}, R_{H}\right)$

where $\left(L_{H}, R_{H}\right)=\left(\min \left(L_{1 H}, L_{2 H}\right), \min \left(L_{1 H}, R_{1 H}\right)\right)$

Step 2. Using Equation 1, find the expectation values of centroid, $M_{1}$ and $M_{2}$ of $\tilde{A}_{1}^{*}$ and $\tilde{A}_{2}^{*}$, respectively.

Step 3. Using Equation 2, find the transfer coefficients, $\lambda_{1}$ and $\lambda_{2}$ of $\tilde{A}_{1}^{*}$ and $\tilde{A}_{2}^{*}$, respectively.

Step 4. Obtain $s^{L}\left(\tilde{A}_{1}^{*}\right)$ and $s^{R}\left(\tilde{A}_{2}^{*}\right)$ by using (3) and (4). 
Step 5. Obtain the values of $s\left(\tilde{A}_{1}^{*}\right)$ and $s\left(\tilde{A}_{2}^{*}\right)$ by using (5)

Step 6. The fuzzy sets with different heights, $\tilde{A}_{1}^{*}$ and $\left(\tilde{A}_{2}^{*}\right)$ can be compared as follows:

$\tilde{A}_{1} \succ \tilde{A}_{2}$ if and only if $s\left(\tilde{A}_{1}^{*}\right) \succ s\left(\tilde{A}_{2}^{*}\right)$

$\tilde{A}_{1} \prec \tilde{A}_{2}$ if and only if $s\left(\tilde{A}_{1}^{*}\right) \prec s\left(\tilde{A}_{2}^{*}\right)$.

$\tilde{A}_{1}: \tilde{A}_{2}$ if and only if $s\left(\tilde{A}_{1}^{*}\right): s\left(\tilde{A}_{2}^{*}\right)$

\section{Comparative study}

In this section, different generalized fuzzy sets and fuzzy sets with different heights are taken to compare the results of the proposed ranking method and the existing ranking method.

Set 1. Let $\tilde{A}=(0.2,0.4,0.6,0.8 ; 0.35)$ and $\widetilde{B}=(0.1,0.2,0.3,0.4 ; 0.7)$ be two generalized fuzzy sets. Use the following steps to compare $\widetilde{A}$ and $\widetilde{B}$ :

Step 1. Transform $\tilde{A}, \widetilde{B}$ into $\widetilde{A}^{*}$ and $\widetilde{B}^{*}$, where $\tilde{A}^{*}=(0.2,0.4,0.6,0.8 ; 0.35)$ and $\widetilde{B}^{*}=(0.1,0.2,0.3,0.4 ; 0.35)$.

Step 2. Using Equation $1, M_{\tilde{A}^{*}}=-3.766$ and $M_{\widetilde{B}^{*}}=1.592$.

Step 3. Using Equation 2, $\lambda_{\widetilde{A}^{*}}=0$ and $\lambda_{\widetilde{B}^{*}}=1$.

Step 4. Using Equations 3 and $4, s^{L}\left(\tilde{A}^{*}\right)=0.07$, $s^{R}\left(\tilde{A}^{*}\right)=0.035 \quad$ and $\quad s^{L}\left(\tilde{B}^{*}\right)=0.175$, $s^{L}\left(\tilde{B}^{*}\right)=0.0175$.
Step 5. Using Equation $5, \quad s\left(\tilde{A}^{*}\right)=0$ and $s\left(\widetilde{B}^{*}\right)=0.0175$

Since $s\left(\tilde{A}^{*}\right) \prec s\left(\widetilde{B}^{*}\right)$, so $\tilde{A} \prec \widetilde{B}$

Set 2. Let $\tilde{A}=(0.1,0.2,0.4,0.5 ; 1)$ and $\widetilde{B}=(0.1,0.3,0.3,0.5 ; 1)$ be two generalized fuzzy sets. Use the following steps to compare $\tilde{A}$ and $\widetilde{B}$ :

Step 1. Transform $\widetilde{A}, \widetilde{B}$ into $\widetilde{A}^{*}$ and $\widetilde{B}^{*}$, where $\tilde{A}^{*}=(0.1,0.2,0.4,0.5 ; 1)$ and $\widetilde{B}^{*}=(0.1,0.3,0.3,0.5 ; 1)$.

Step 2. Using Equation 1, $M_{\widetilde{A}^{*}}=0.18771$ and $M_{\widetilde{B}^{*}}=0.10115$.

Step 3. Using Equation 2, $\lambda_{\widetilde{A}^{*}}=1$ and $\lambda_{\widetilde{B}^{*}}=0$.

Step 4. Using Equations 3 and $4, s^{L}\left(\tilde{A}^{*}\right)=0.5$, $s^{R}\left(\tilde{A}^{*}\right)=0.2$ and $s^{L}\left(\tilde{B}^{*}\right)=0.1, s^{L}\left(\tilde{B}^{*}\right)=0.2$.

Step 5. Using Equation 5, $s\left(\tilde{A}^{*}\right)=0.5$ and $s\left(\tilde{B}^{*}\right)=0$

Since $s\left(\tilde{A}^{*}\right) \succ s\left(\widetilde{B}^{*}\right)$, so $\tilde{A} \succ \widetilde{B}$

Set 3. Let $\tilde{A}=(1,2,3,4 ; 0.6,0.4) \quad$ and $\widetilde{B}=(0,3,4,5 ; 0.4,0.2)$ be two fuzzy sets with different heights. Use the following steps to compare $\tilde{A}$ and $\tilde{B}$ :

Step 1. Transform $\tilde{A}, \widetilde{B}$ into $\widetilde{A}^{*}$ and $\widetilde{B}^{*}$, where $\tilde{A}^{*}=(1,2,3,4 ; 0.4,0.2)$

and $\widetilde{B}^{*}=(0,3,4,5 ; 0.4,0.2)$.

Step 2. Using Equation 1, $M_{\tilde{A}^{*}}=-4.78$ and $M_{\widetilde{B}^{*}}=-2.50$. 
Step 3. Using Equation 2, $\lambda_{\widetilde{A}^{*}}=0$ and $\lambda_{\widetilde{B}^{*}}=1 . \quad \underset{\sim}{\text { Set }} 4 . \quad$ Let $\quad \tilde{A}=(2,5,6,7 ; 0.6,0.4) \quad$ and $\widetilde{B}=(3,4,5,6 ; 0.8,0.6)$ be two fuzzy sets with

Step 4. Using Equations 3 and $4, s^{L}\left(\tilde{A}^{*}\right)=0.6$, $s^{R}\left(\tilde{A}^{*}\right)=0.3$ and $s^{L}\left(\tilde{B}^{*}\right)=0.6, s^{L}\left(\tilde{B}^{*}\right)=0.1$.

different heights. Use the following steps to compare $\tilde{A}$ and $\tilde{B}$ :

Step 1. Transform $\tilde{A}, \widetilde{B}$ into $\widetilde{A}^{*}$ and $\widetilde{B}^{*}$, where

Step 5. Using Equation 5, $s\left(\tilde{A}^{*}\right)=0$ and $s\left(\tilde{B}^{*}\right)=0.6$

Since $s\left(\tilde{A}^{*}\right) \prec s\left(\tilde{B}^{*}\right)$, so $\tilde{A} \prec \widetilde{B}$

$\tilde{A}^{*}=(2,5,6,7 ; 0.6,0.4)$ and $\widetilde{B}^{*}=(3,4,5,6 ; 0.6,0.4)$.

Step 2. Using Equation 1, $M_{\tilde{A}^{*}}=23.83$ and $M_{\widetilde{B}^{*}}=34.87$.
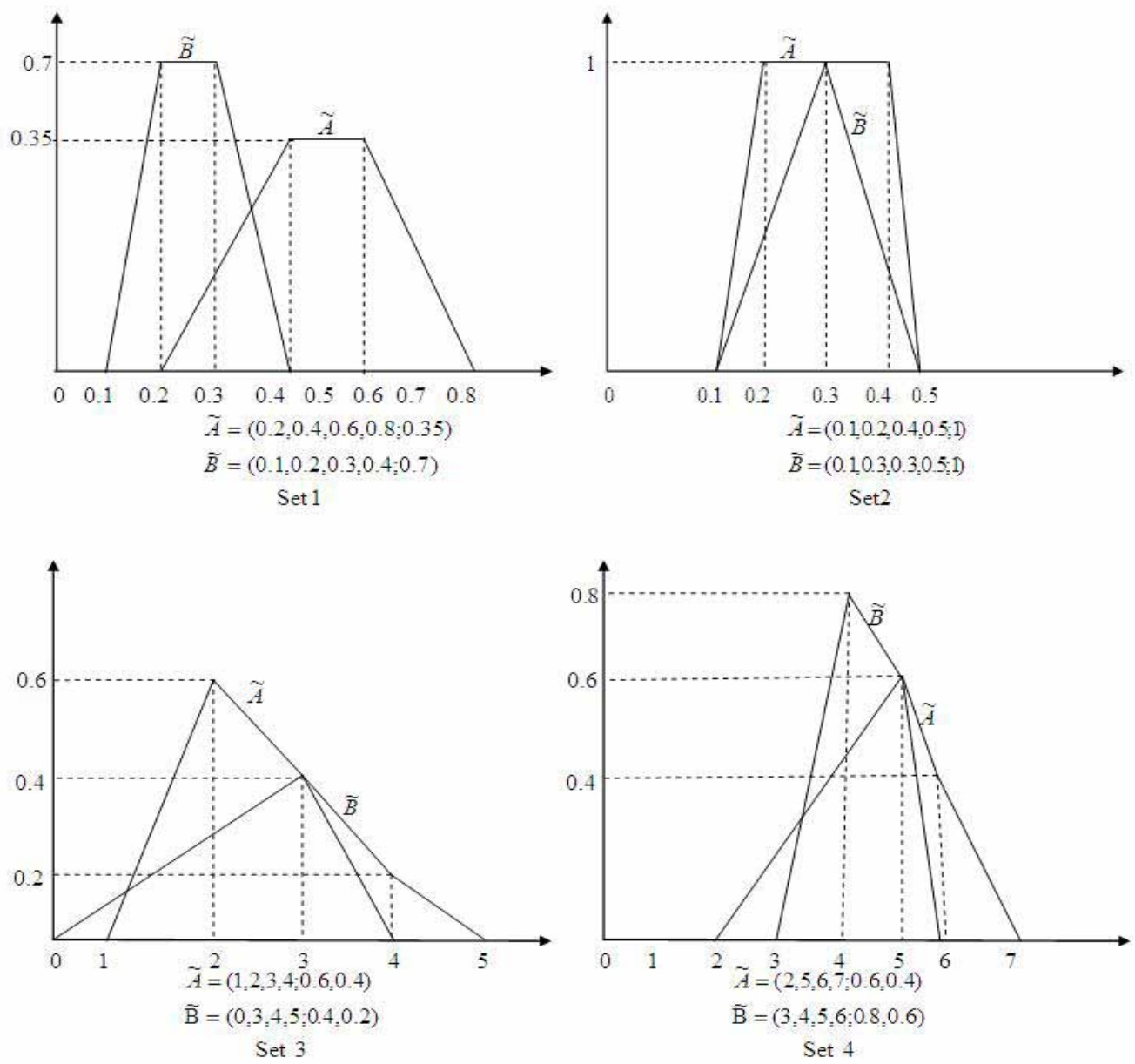

Figure 3. Generalized fuzzy sets and fuzzy sets witth diferent heights. 
Step 3. Using Equation 2, $\lambda_{\widetilde{A}^{*}}=0$ and $\lambda_{\widetilde{B}^{*}}=1$.

Step 4. Using Equations 3 and $4, s^{L}\left(\tilde{A}^{*}\right)=0.9$, $s^{R}\left(\tilde{A}^{*}\right)=0.2$ and $s^{L}\left(\tilde{B}^{*}\right)=0.9, s^{L}\left(\tilde{B}^{*}\right)=0.6$.

Step 5. Using Equation $5, s\left(\tilde{A}^{*}\right)=0$ and $s\left(\tilde{B}^{*}\right)=0.9$

Since $s\left(\tilde{A}^{*}\right) \prec s\left(\widetilde{B}^{*}\right)$, so $\tilde{A} \prec \widetilde{B}$

\begin{tabular}{|c|c|c|c|c|}
\hline Methods & Set 1 & Set 2 & Set 3 & Set 4 \\
\hline Cheng [10] & $A \prec B$ & $A: B$ & N.A & N.A \\
\hline $\begin{array}{l}\text { Chu and } \\
\text { Tsao [11] }\end{array}$ & $\tilde{A} \prec \widetilde{B}$ & $\tilde{A}: \widetilde{B}$ & N.A & N.A \\
\hline $\begin{array}{c}\text { Chen and } \\
\text { Chen [5] }\end{array}$ & $\widetilde{A} \prec \widetilde{B}$ & $\widetilde{A} \prec \widetilde{B}$ & N.A & N.A \\
\hline $\begin{array}{c}\text { Abbasbandy } \\
\text { and Hajjari } \\
{[1]}\end{array}$ & N.A & $\tilde{A}: \widetilde{B}$ & N.A & N.A \\
\hline $\begin{array}{l}\text { Chen and } \\
\text { Chen [6] }\end{array}$ & $\tilde{A} \prec \widetilde{B}$ & $\tilde{A} \prec \widetilde{B}$ & N.A & N.A \\
\hline $\begin{array}{c}\text { Liou and } \\
\text { Wang [17] }\end{array}$ & $\tilde{A} \prec \widetilde{B}$ & $\tilde{A}: \widetilde{B}$ & N.A & N.A \\
\hline $\begin{array}{l}\text { Lee and } \\
\text { Chen [16] }\end{array}$ & $\widetilde{A} \prec \widetilde{B}$ & $\tilde{A} \succ \widetilde{B}$ & $\tilde{A} \prec \widetilde{B}$ & $\tilde{A} \succ \widetilde{B}$ \\
\hline $\begin{array}{c}\text { Rommelfang } \\
\text { er [20] }\end{array}$ & N.A & $\widetilde{A}: \widetilde{B}$ & N.A & N.A \\
\hline $\begin{array}{l}\text { Proposed } \\
\text { approach }\end{array}$ & $\widetilde{A} \prec \widetilde{B}$ & $\tilde{A} \succ \widetilde{B}$ & $\tilde{A} \prec \tilde{B}$ & $\tilde{A} \prec \widetilde{B}$ \\
\hline
\end{tabular}

Table 1. Comparison of the proposed ranking approach with the existing ranking approach.

Note:- where N.A denotes the "not applicable".

\section{Conclusions}

In this paper, with the help of counterexamples, it is proved that the ranking method proposed by Lee and Chen [16] is incorrect. A new approach for the ranking of fuzzy sets with different heights, as well as the results of the proposed ranking method and the existing ranking method are compared.

\section{Acknowledgements}

The authors would like to thank the Editor-in-Chief and the anonymous referees for the various suggestions which have led to an improvement in both the quality and clarity of the paper.

\section{References}

[1] S. Abbasbandy and T. Hajjari, "A new approach for ranking of trapezoidal fuzzy numbers", Computers and Mathematics with Applications, 57, 413-419, 2009.

[2] B. Assady, "The revised method of ranking LR fuzzy number based on deviation degree", Expert Systems with Applications, 37, 5056-5060 2010.

[3] B. Assady, "Revision of distance minimization method for ranking of fuzzy numbers", Applied Mathematical Modelling, 35, 1306-1313, 2011

[4] L.M. Campos and A. Gonzalez, "A subjective approach for ranking fuzzy numbers", Fuzzy Sets and Systems, 29, 145-153, 1989.

[5] S. J. Chen and S. M. Chen, "Fuzzy risk analysis based on the ranking of generalized trapezoidal fuzzy numbers", Applied Intelligence, 26, 1-11, 2007.

[6] S. M. Chen and J. H. Chen, "Fuzzy risk analysis based on ranking generalized fuzzy numbers with different heights and different spreads", Expert Systems with Applications, 36, 6833-6842, 2009.

[7] S. M. Chen et al., "Fuzzy risk analysis based on ranking generalized fuzzy numbers with different left heights and right heights", Expert Systems with Applications, 39, 6320-6334, 2012

[8] C.C. Chen and H.C. Tang, "Ranking nonnormal pnorm trapezoidal fuzzy numbers with integral value", Computers and Mathematics with Applications, 56, 23402346, 2008. 
[9] S.M. Chen and C.H. Wang, "Fuzzy risk analysis based on ranking fuzzy numbers using $\alpha-$ cuts, belief features and signal/noise ratios", Expert Systems with Applications, 36, 5576-5581, 2009.

[10] C.H. Cheng, "A new approach for ranking fuzzy numbers by distance method", Fuzzy Sets and Systems, 95, 307-317, 1998.

[11] T. C. Chu and C. T. Tsao, "Ranking fuzzy numbers with an area between the centroid point and original point", Computers and Mathematics with Applications, 43, 111-117, 2002.

[12] Y. Deng and Q. Liu, "A TOPSIS-based centroidindex ranking method of fuzzy numbers and its applications in decision making", Cybernetics and Systems, 36, 581-595, 2005.

[13] R. Ezzati et al. , "An approach for ranking of fuzzy numbers", Expert Systems with Applications, 39, 690-695, 2012.

[14] R. Jain, "Decision-making in the presence of fuzzy variables", IEEE Transactions on Systems Man and Cybernetics, 6, 698-703, 1976.

[15] H.C. Kwang and J.H. Lee, "A method for ranking fuzzy numbers and its application to decision making", IEEE Transactions on Fuzzy Systems, 7, 677-685, 1999.

[16] W.E. Lee and S.M. Chen, "Fuzzy risk analysis based on fuzzy numbers with different shapes and different deviations", Expert Systems with Applications, 34, 27632771, 2008.

[17] T. S. Liou and M. J. Wang, "Ranking fuzzy numbers with integral value", Fuzzy Sets and Systems, 50, 247-255, 1992.

[18] M. Modarres and S. Sadi-Nezhad, "Ranking fuzzy numbers by preference ratio", Fuzzy Sets and Systems, 118, 429-436, 2001.

[19] N. Ramli and D. Mohamad, "A comparative analysis of centroid methods in ranking fuzzy numbers", European Journal of Scientific Research, 28, 492-501, 2009.

[20] H. Rommelfanger, "A general concept for solving linear multicriteria programming problems with crisp, fuzzy or stochastic values", Fuzzy Sets and Systems, 158, 1892-1904, 2007.

[21] X. Wang and E.E. Kerre, "Reasonable properties for the ordering of fuzzy quantities (I)", Fuzzy Sets and Systems, 118, 375-385, 2001.
[22] Y.J. Wang and H.S. Lee, "The revised method of ranking fuzzy numbers with an area between the centroid and original points", Computers and Mathematics with Applications, 55, 2033-2042, 2008.

[23] R.R. Yager, "A procedure for ordering fuzzy subsets of the unit interval", Information Sciences 24, 143-161, 1981.

[24] L.A. Zadeh, "Fuzzy sets", Information and Control, 8, 338-353 1965. 